\title{
Tuberculosis ganglionar periférica en pediatría: 16 años de experiencia en un centro pediátrico de tercer nivel de Buenos Aires, Argentina
} Peripheral tuberculous lymphadenitis in pediatrics: 16 years of experience in a tertiary care pediatric hospital of Buenos Aires, Argentina

\author{
Dra. Silvina E. Neyro ${ }^{a}$ Dra. Isabel R. Squassi ${ }^{a}$, Dr. Martín Medín ${ }^{b}$ \\ Bioq. Ana Caratozzolo ${ }^{c}$ Dr. Andrés Martínez Burkett y Dra. María C. Cerqueiro ${ }^{a}$
}

\section{RESUMEN}

La tuberculosis extrapulmonar representa el 15$20 \%$ de todas las formas de presentación. La tuberculosis ganglionar periférica es la segunda forma extrapulmonar más frecuente en niños de Argentina, luego de la pleural.

En el Servicio de Tisiología del Hospital de Niños "Dr. Ricardo Gutiérrez", se analizaron en forma retrospectiva 92 casos de tuberculosis ganglionar periférica asistidos entre agosto, 2000-septiembre, 2015. La edad media fue $8,7 \pm 5$ años. Las adenopatías fueron periféricas únicas $(31,5 \%)$, periféricas múltiples $(20,6 \%)$ y periféricas asociadas a profundas $(47,8 \%)$. Predominóla localización cervical $(80 \%)$. El $80 \%$ recibió antibioticoterapia previa, sin respuesta. El $56 \%$ tenía foco de contagio conocido; $69 \%$, prueba cutánea de tuberculina positiva y $54 \%$, radiografía de tórax patológica. Todos iniciaron tratamiento antifímico por clínica compatible, exposición y/o prueba cutánea de tuberculina positiva, antes de la confirmación microbiológica o histológica. La evolución fue curación $(81,5 \%)$, derivación cercana al domicilio $(8,7 \%)$, abandono $(8,7 \%)$. Un paciente falleció. Palabras clave: Mycobacterium tuberculosis, tuberculosis ganglionar, linfadenopatía periférica,

a. Sección de Tisiología.

b. Servicio de Anatomía Patológica.

c. Laboratorio de Biología Molecular.

d. Sección Dermatología. Hospital de Niños Ricardo Gutiérrez. Ciudad de Buenos Aires.

Correspondencia:

Dra. Silvina E. Neyro:

silvinaneyro@gmail.com

Financiamiento:

Ninguno.

Conflicto de intereses:

Ninguno que declarar.

Recibido: 22-12-2017

Aceptado: 16-5-2018 de las diez primeras causas mundiales de mortalidad. ${ }^{1}$

A pesar de que la lucha contra la tuberculosis ha tenido un progreso significativo -43 millones de vidas han sido salvadas desde el año 2000 gracias a los esfuerzos realizados para optimizar el diagnóstico y tratamiento-, la batalla dista de estar siendo ganada, ya que, todos los días, más de 4000 personas mueren en el mundo a causa de esta enfermedad. Se estima que, en 2015, 1 millón de niños enfermaron y 170000 niños murieron por tuberculosis (sin incluir a los niños que vivían con virus de la inmunodeficiencia humana-VIH-). ${ }^{2}$

En Argentina, se diagnosticaron 9761 casos (nuevos más recaídas) de tuberculosis $(22,6$ casos $/ 100000$ habitantes) en 2015. Representó 156 casos más que en 2014. Si bien la ocurrencia nacional de tuberculosis en la edad pediátrica y la adolescencia fue decreciente, la tendencia jurisdiccional fue desigual. Al comparar la notificación de casos de tuberculosis entre 1990 y 2015, en menores de 9 años, se observa una reducción. Sin embargo, la tendencia prácticamente estable de la notificación de casos en adolescentes de 15 a 19 años durante los últimos 16 años resulta preocupante y requiere fortalecer las acciones de control. ${ }^{3}$

La tuberculosis extrapulmonar representa, aproximadamente, el 15-20\% de todas las formas de presentación. ${ }^{4,5}$ La tuberculosis ganglionar periférica (TGP) es, a su vez, una de las formas más frecuentes de presentación extrapulmonar 
en pediatría y se encuentra en segundo lugar luego de las pleurales en nuestro país. ${ }^{3}$ Puede acompañarse o no de compromiso pulmonar activo concomitante. La localización cervical ha sido descrita tradicionalmente como su ubicación más frecuente (60-80\%), antes conocida como "escrófula". Sin embargo, otros grupos ganglionares pueden verse afectados. ${ }^{5}$ Dada la diversidad de diagnósticos diferenciales de adenopatías en pediatría y/o la ausencia de sospecha clínica precoz, la TGP es un desafío permanente para el diagnóstico y tratamiento oportuno.

En nuestro conocimiento, este estudio representa la primera casuística publicada sobre TGP en pediatría en nuestro país, en los últimos años. El objetivo fue evaluar el comportamiento de los casos de TGP diagnosticados en nuestro Servicio, abordando aspectos demográficos, epidemiológicos, clínicos, diagnósticos, terapéuticos y evolutivos.

\section{POBLACIÓN Y MÉTODOS}

Estudio retrospectivo, descriptivo de los casos de TGP asistidos en el Servicio de Tisiología del Hospital de Niños "Dr. Ricardo Gutiérrez" (hospital pediátrico de tercer nivel de la Ciudad Autónoma de Buenos Aires, Argentina) en el período comprendido entre agosto de $2000 \mathrm{y}$ septiembre de 2015.

Los datos demográficos, clínicos (comorbilidades, signos y síntomas, formas de presentación), epidemiológicos (fuente de contagio), diagnósticos (estudios de laboratorio, prueba de reacción a la tuberculina, estudios microbiológicos y de diagnóstico por imágenes), terapéuticos (tiempo hasta el inicio de tratamiento, toxicidad) y evolutivos (curación, abandono o muerte) se obtuvieron del análisis de las historias clínicas de los pacientes con diagnóstico confirmado o sospechoso de TGP y se recolectaron en una base de datos destinada a tal fin. Para el análisis estadístico de los datos, se utilizó el programa Epi Info $7^{\circledR}$. Las variables continuas se informaron como media o medianas y rango. Las variables categóricas se expresaron como número y porcentaje.

El presente estudio fue evaluado y aprobado por el Comité de Ética del Hospital.

\section{RESULTADOS}

En el período analizado, se realizó el diagnóstico, seguimiento y tratamiento de 1607 casos pediátricos totales de tuberculosis, con un promedio de 100,4 casos por año. El $24 \%$ (381 pacientes) presentaron compromiso extrapulmonar. De estos, 92 casos fueron TGP, lo cual representó el $24 \%$ de las tuberculosis extrapulmonares y el $6 \%$ del total de los casos de tuberculosis. La distribución de casos por cada año comprendido en el análisis demostró que el $51 \%$ (47/92) se concentró en los últimos 5 años (2011-2015) (Figura 1).

El análisis de datos demográficos de estos 92 casos pediátricos de TGP se muestra en la Tabla 1. La edad media de presentación fue $8,7 \pm 5$ años; el 42,4 \% del total de los niños pertenecían al grupo de mayores de 10 años (Figura 2). Solo un paciente fue mayor de 18 años; tenía seguimiento multidisciplinario en nuestro Hospital desde su infancia, por diagnóstico de lupus eritematoso sistémico. Predominó el sexo femenino, y las mujeres estuvieron afectadas a mayor edad respecto de los varones (media de edad en mujeres: 10 años vs. media de edad en varones: 7 años; $p<0,04)$. El $59 \%$ de los casos ocurrieron en niños sin patologías preexistentes $\mathrm{y} / \mathrm{o}$ asociadas (Tabla 1).

Las adenopatías fueron periféricas únicas en el $31,5 \%$ de los casos (29/92), periféricas múltiples en el 20,6 \% (19/92) y el 47,8 \% (44/92) presentó alguna adenopatía profunda asociada. Respecto de la localización, predominaron las adenopatías cervicales $(80 \%)$, seguidas en frecuencia por las faciales $(12 \%)$, axilares $(11 \%)$, supraclaviculares $(8 \%)$ e inguinales (5\%).

Al momento de la consulta, el $49 \%$ (45/92) de los pacientes presentaba una adenopatía de evolución subaguda o crónica, sin signosintomatología acompañante. Otros signos y síntomas, como fiebre, dificultad respiratoria, síndrome de impregnación y pérdida de peso, estuvieron presentes en algunos pacientes (Figura 3). El $80 \%$ de los casos recibió algún esquema antibiótico para gérmenes comunes antes de la consulta o derivación a nuestro Servicio, sin respuesta clínica.

Respecto al diagnóstico, solo el $56 \%$ de los casos tenía una fuente de contagio conocida, y fue más frecuente la identificación del foco a menor edad (media de edad de los pacientes con foco conocido: 7 años vs. foco no conocido/ sospechoso: 11 años; $\mathrm{p}<0,006$ ). El $100 \%$ de los pacientes estaban vacunados con bacilo de Calmette-Guérin (BCG). La prueba cutánea de tuberculina (purified protein derivative; PPD, por sus siglas en inglés) resultó positiva en el $69 \%$ de los casos y negativa en el $31 \%$ restante. 
E1 $54 \%$ de los casos presentó radiografía de tórax patológica (patrones radiológicos más frecuentes: ensanchamiento hilio-mediastinal y neumonía). Los hallazgos de laboratorio fueron inespecíficos (media de recuento de glóbulos blancos: 9440 células $/ \mathrm{mm}^{3} \pm 4600$; media de velocidad de eritrosedimentación globular: $45 \mathrm{~mm} / \mathrm{h} \pm 32$; entre otros). La ecografía resultó de utilidad para el control evolutivo de la lesión y para determinar su accesibilidad para realizar procedimientos quirúrgicos diagnósticos en 70 pacientes $(76 \%)$

FIGURA 1. Casos de tuberculosis ganglionar periférica asistidos por año (período 2000-2015)

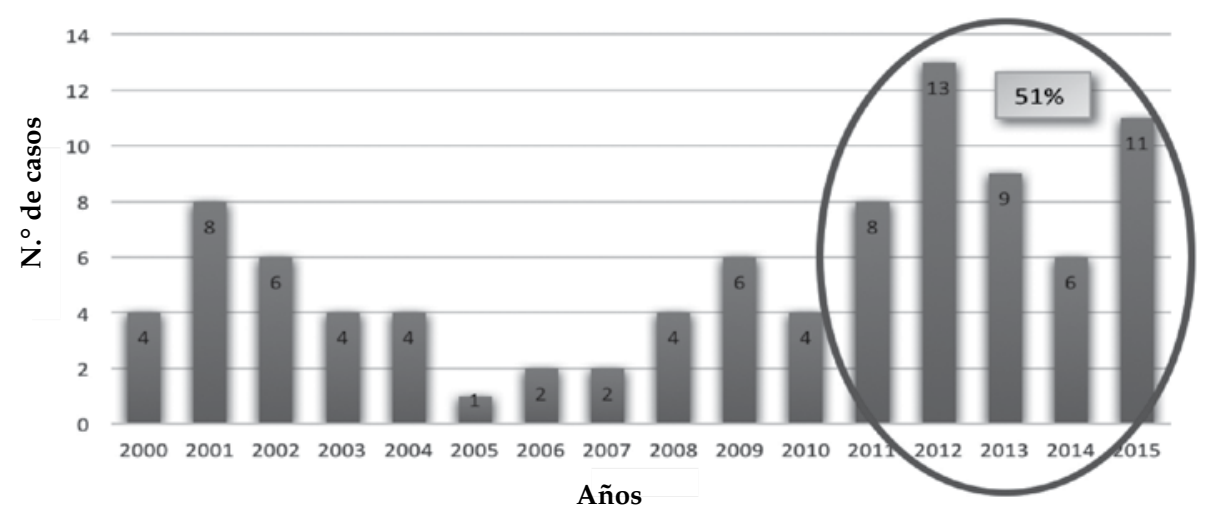

FIGURA 2. Distribución de casos de tuberculosis ganglionar periférica por grupos etarios y sexo

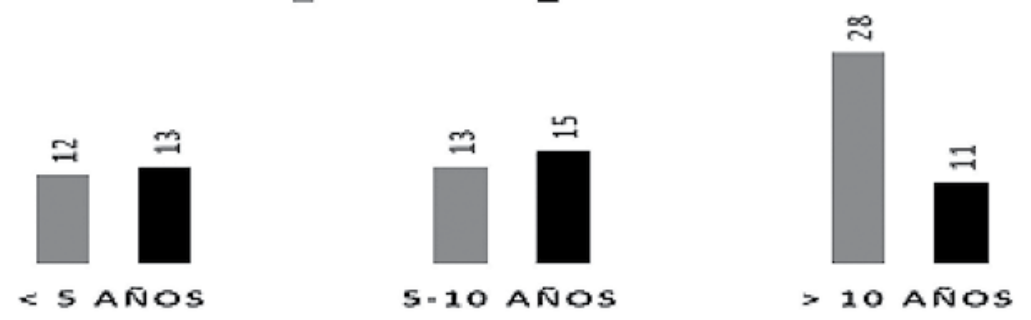

TABLA 1. Características demográficas y comorbilidades de la población en estudio

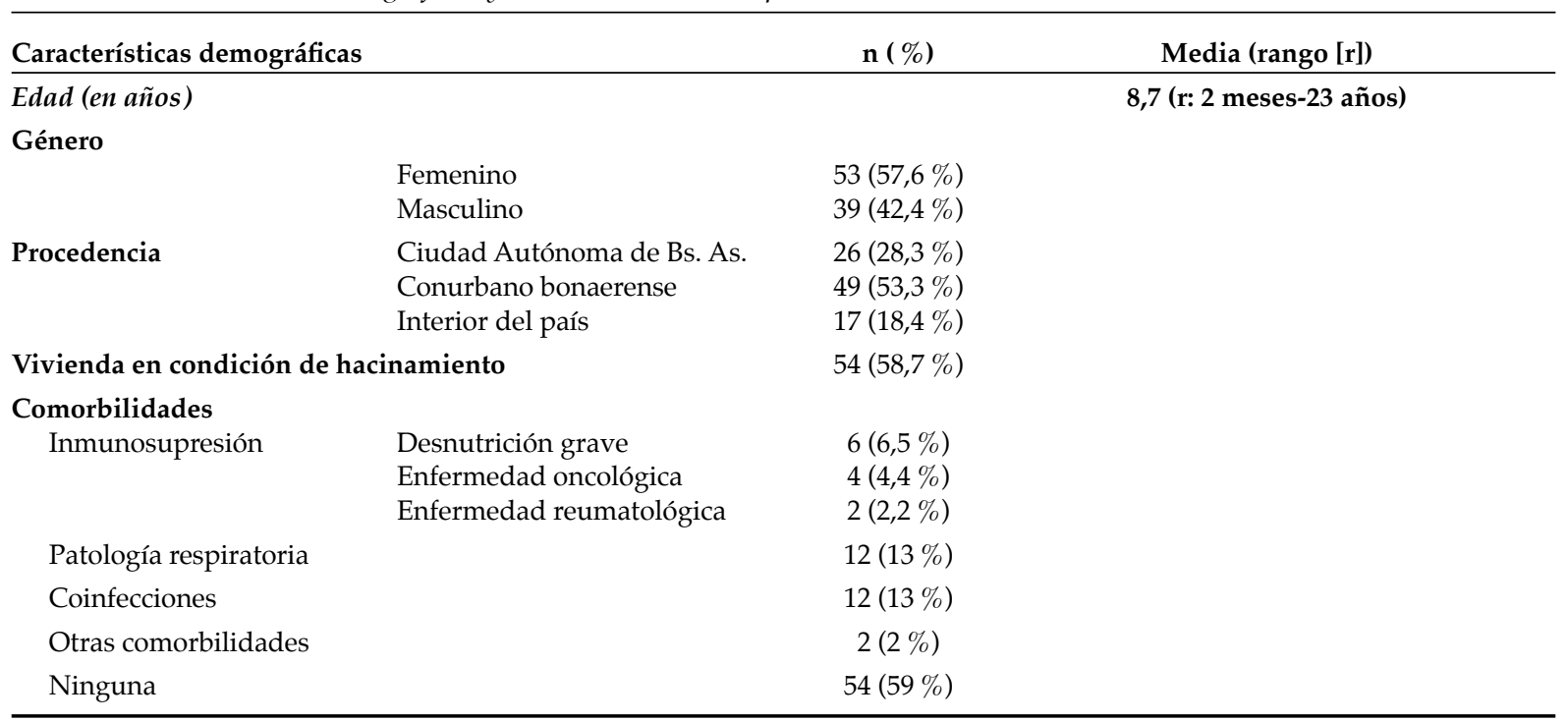


El $63 \%(58 / 92)$ de los pacientes requirió internación (ya sea para completar estudios o por gravedad), con una media de 14 días. Esta fue más prolongada en huéspedes inmunocomprometidos (media de 39 días, $p<0,001$ ), presencia de foco pulmonar (media de 25 días, $\mathrm{p}<0,001$ ) y reacción adversa a fármacos antituberculosos (RAFA) (media de 25 días, $\mathrm{p}<0,01$ ).

Un total de 84/92 (91\%) fueron estudiados para tuberculosis, ya sea a partir de muestras ganglionares, de secreciones respiratorias o ambas mediante examen directo, cultivo, prueba de reacción en cadena de la polimerasa (polymerase chain reaction; PCR, por sus siglas en inglés) convencional con primers para el segmento de inserción IS6110 y/o histología. Se realizaron 77 procedimientos quirúrgicos diagnósticos a nivel ganglionar: 26/92 por punción y aspiración con aguja fina (PAAF), 46/92 por biopsia y 5/92 por exéresis. Se confirmó el diagnóstico microbiológicamente en el $60 \%$ de las muestras totales (respiratorias $\mathrm{y} / \mathrm{o}$ ganglionares) estudiadas por cultivo y el $64,7 \%$ de las muestras analizadas por PCR (Tabla 2). Todos los cultivos positivos fueron cepas de
Mycobacterium tuberculosis sensibles a drogas de primera línea. En ocho pacientes, no se pudieron realizar estos estudios diagnósticos por falta de secreciones respiratorias y/o de disponibilidad hospitalaria para efectivizar el procedimiento quirúrgico diagnóstico en forma mediata; sin embargo, todos ellos presentaron una respuesta favorable al tratamiento empírico instaurado.

Todos los pacientes iniciaron tratamiento (3 o 4 drogas antifímicas orales de primera línea: isoniacida, rifampicina, pirazinamida, con o sin etambutol) por un cuadro clínico compatible, exposición y/o PPD positiva, antes de la confirmación microbiológica o histológica. La media de tiempo de evolución de las adenopatías al momento del inicio del tratamiento antifímico fue de 82 días $\pm 70,5$ (mayor en niños inmunocompetentes [ $p<0,05]$ y en adenopatías múltiples [p <0,02]). El 19,5\% presentaron RAFA; la presencia de hepatitis fue el hallazgo más frecuente $(9 / 18)$.

La evolución fue favorable en el 81,5\% de los casos, que lograron la curación. El 8,7\% fue derivado en el curso del tratamiento para completarlo en centros de salud cercanos al

FIGURA 3. Signos y sintomas acompañantes de la adenopatía subaguda o crónica

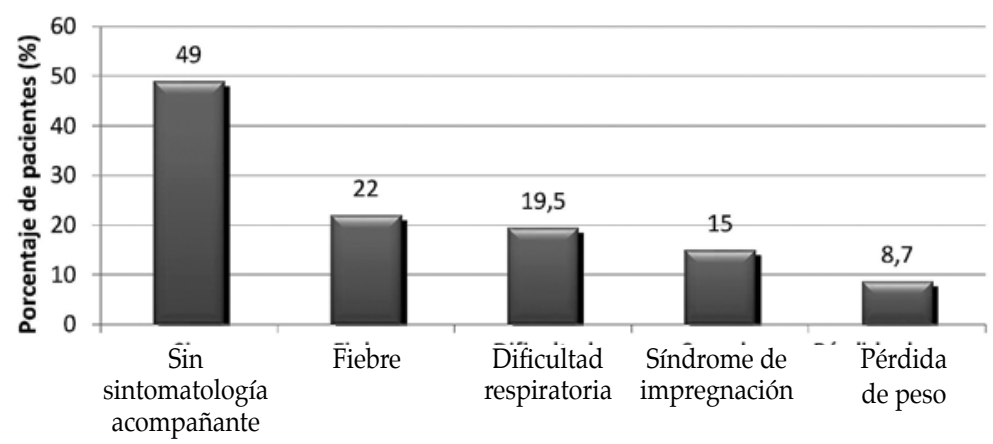

TABLA 2. Porcentaje de positividad para Mycobacterium tuberculosis, según la muestra analizada

$\left.\begin{array}{lc}\begin{array}{l}\text { Estudios realizados en muestras } \\ \text { ganglionares y/o respiratorias }\end{array} & \begin{array}{c}\text { Muestras positivas/total de las analizadas } \\ \text { ( } \% \text { de positivos) }\end{array} \\ \hline \text { Examen directo (baciloscopía) } & \begin{array}{c}7 / 84(8,3 \%) \\ \text { Cultivo }\end{array} \\ & \begin{array}{c}\text { Las } 7 \text { muestras positivas fueron } 4 \text { ganglionares, } 3 \text { respiratorias. } \\ 42 / 60(60 \%)\end{array} \\ \text { PCR } & \text { Las } 42 \text { muestras positivas fueron } 21 \text { ganglionares, } 6 \text { respiratorias y } \\ 15 \text { ganglionares }+ \text { secreciones respiratorias. } \\ 22 / 34(64,7 \%)\end{array}\right)$

PCR: reacción en cadena de la polimerasa. 
domicilio. Otro 8,7\% abandonó el tratamiento. Solo un paciente, con diagnóstico concomitante de síndrome linfoproliferativo, falleció. En 26 pacientes, se continuó el tratamiento en forma empírica por alto riesgo epidemiológico y clínica compatible, con respuesta favorable en el $81 \%$ de los casos.

\section{DISCUSIÓN}

Nuestro estudio contempla 16 años de casuística pediátrica sobre adenitis tuberculosa. Esta forma de presentación de tuberculosis extrapulmonar constituyó la segunda en frecuencia luego de la pleural durante todo el período; sin embargo, fue reportada por otros estudios como la primera forma clínica de tuberculosis extrapulmonar en otros países. ${ }^{6,7}$

La adenitis periférica de causa tuberculosa puede presentarse en el contexto de una primoinfección (como puede observarse en niños pequeños y/o en pacientes inmunocomprometidos) o puede reflejar la reactivación de una infección primaria previa. La epidemiología de la TGP varía según se trate de países desarrollados o países en desarrollo. En los países desarrollados, la mayoría de los casos de TGP se producen entre los adultos inmigrantes de países con tuberculosis endémica, ${ }^{8,9}$ que presentan un patrón consistente con enfermedad por reactivación. Esto fue documentado en series de casos de TGP de países como Francia y Alemania, en las que alrededor del $70 \%$ de los casos ocurrieron en inmigrantes. ${ }^{8,9} \mathrm{Si}$ bien la TGP se consideraba clásicamente una enfermedad de la infancia, en los últimos años, la edad máxima de inicio en los países desarrollados ha migrado de la infancia a los adultos jóvenes (de 20 a 40 años). ${ }^{8,10}$

Como en la mayoría de las series publicadas, se evidenció que la TGP fue más común entre las mujeres, ${ }^{7,11}$ un patrón distinto al de la tuberculosis pulmonar, enfermedad que suele ser más común entre los hombres. ${ }^{12}$

La región cervicofacial fue el área de localización más frecuente de las adenopatías, lo que coincidió con otras publicaciones. ${ }^{5}$ Suelen presentarse como adenopatías indoloras de progresión lenta. ${ }^{13} \mathrm{El}$ drenaje espontáneo está descrito hasta en el 4-11\% de los casos. ${ }^{13,14}$ Los signos y síntomas sistémicos pueden no estar presentes, si bien se reportan hasta en el $55 \%$ de los casos de adenitis tuberculosa ${ }^{15} \mathrm{y}$ deben ser tenidos en cuenta al momento del interrogatorio. En nuestro estudio, el $51 \%$ de los casos presentó algún signo o síntoma acompañante.
La PPD resultó positiva en el $69 \%$ de nuestros casos. Un estudio llevado a cabo por A. Polesky et al., en Estados Unidos evidenció un resultado positivo en la prueba de la tuberculina en 90 de 92 (98\%) pacientes inmunocompetentes con adenitis por tuberculosis. ${ }^{16}$ En estudios de linfadenitis tuberculosa realizados en Corea del Sur e India, la sensibilidad de la PPD fue del $86 \%$ y $89 \%$, respectivamente. ${ }^{17,18}$

Se detectaron alteraciones radiológicas compatibles con la presencia de adenopatías hilio-mediastinales o lesiones pulmonares en el $54 \%$ de nuestros casos, lo que correspondió a una proporción mayor que la reportada por otras series. ${ }^{19,20}$ La presencia de lesión pulmonar asociada fue discrepante en las distintas publicaciones: M. S. Kamal et al., la reportaron únicamente en el 3,1\% de los casos; ${ }^{11} \mathrm{~B}$. C. Jha et al. y P. B. Magsi et al., refirieron su presencia en el $16 \%$ y $7,5 \%$, respectivamente. ${ }^{21,22} \mathrm{Sin}$ embargo, estudios como el de N. Choudhury et al., evidenciaron compromiso pulmonar en $48 \%$ de los casos, ${ }^{23}$ resultado más acorde con nuestro hallazgo.

En nuestra serie, la demora diagnóstica y terapéutica se atribuyó al bajo índice de sospecha clínica y epidemiológica, pues se interpretaron los síntomas como adenitis piógena en la mayoría de los casos y no se tuvo en cuenta la ausencia de respuesta al tratamiento antibiótico o el antecedente de fuente conocida de contagio, dato epidemiológico esencial para la sospecha diagnóstica. ${ }^{24}$

En cuanto al agente etiológico, M. bovis ha sido históricamente un patógeno frecuente en adenitis crónicas; sin embargo, los programas de pasteurización de la leche y control de la tuberculosis bovina casi han eliminado esta fuente de infección humana en los países desarrollados. Si bien, en la actualidad, M. tuberculosis es la causa más frecuente de linfadenitis tuberculosa, ${ }^{25} \mathrm{el}$ consumo de leche no pasteurizada sigue siendo un factor de riesgo para considerar. ${ }^{26}$

La obtención de material para el estudio microbiológico y anatomopatológico a través de PAAF y/o de biopsia ganglionar es fundamental para certificar el diagnóstico. La PAAF ha sido propuesta en los últimos años como una técnica de diagnóstico de primera línea, especialmente, en países endémicos, donde ha demostrado ser sensible y específica. ${ }^{27,28}$ Entre las ventajas que presenta este procedimiento, se destaca que es una intervención segura, costo-efectiva y ambulatoria, además de ser menos invasiva y más 
accesible que la biopsia, sobre todo, en países de recursos limitados. ${ }^{29}$

El diagnóstico de certeza de adenitis tuberculosa puede realizarse a través de cultivo o PCR, mediante la demostración de la presencia de $M$. tuberculosis en material obtenido de un ganglio linfático afectado. El cultivo sigue siendo el estándar de oro para el diagnóstico; sin embargo, su resultado puede demorar semanas. Las características histológicas (ej.: granulomas caseificantes) son muy sugestivas y apoyan fuertemente el diagnóstico en pacientes con clínica compatible y epidemiología positiva para tuberculosis, especialmente, en pediatría, ya que esta población suele caracterizarse por presentar lesiones paucibacilares. En nuestro estudio, la histopatología tuvo un rol diagnóstico importante; se presentaron hallazgos compatibles con tuberculosis en la gran mayoría de las muestras analizadas.

La antibioticoterapia con drogas antifímicas sigue siendo el pilar fundamental del tratamiento frente a una adenitis tuberculosa, si bien la respuesta puede ser lenta e incluso paradojal hasta en el $20 \%$ de los casos (aumento del tamaño de la adenopatía existente o aparición de nuevas adenopatías durante un tratamiento eficaz e incluso después). ${ }^{16,30,31}$ Seis meses de tratamiento suelen ser suficientes para su curación ante bacilos sensibles a drogas de primera línea. Esta recomendación se fundamenta en estudios que no mostraron diferencias significativas entre 6 y 9 meses de tratamiento respecto a tasas de curación (89\%-94 \%) $)^{32,33}$ o de recaída (3\%). ${ }^{34}$

La guía de tratamiento de la Sociedad Americana de Enfermedades Infecciosas (Infectious Diseases Society of America) no recomienda la escisión terapéutica del ganglio, excepto en circunstancias inusuales. ${ }^{35}$ En nuestro caso, se realizó exéresis ganglionar en 5 pacientes; sin embargo, la evolución clínica no se vio modificada por haber sido sometidos a este procedimiento.

Este estudio presenta como fortaleza el poder ofrecer datos relacionados con el seguimiento de un número significativo de pacientes con TGP por un período prolongado, en un mismo centro de atención. Sin embargo, presenta las debilidades inherentes a un estudio retrospectivo de los casos.

\section{CONCLUSIONES}

Es necesario elevar el índice de sospecha de tuberculosis en niños que se presentan con linfadenopatías, en especial, ante una evolución subaguda o crónica de esta, con ausencia de respuesta al tratamiento antibiótico habitual o antecedente de un foco conocido de contagio, dato epidemiológico esencial para ser considerado frente a esta situación.

Optimizar la oportunidad para la toma de muestra es fundamental dada la elevada confirmación diagnóstica que puede obtenerse ante el correcto estudio de los casos.

La TGP es una enfermedad de buen pronóstico aún con un diagnóstico tardío, con alta tasa de curación.

\section{REFERENCIAS}

1. World Health Organization. Tuberculosis. [Acceso: 31 de enero de 2017]. Disponible en: http:/ / www.who.int/ mediacentre/factsheets/fs104/en/.

2. World Health Organization. Global Tuberculosis Report 2016. [Acceso: 1 de febrero de 2017]. Disponible en: http: / / www.who.int/tb/publications/global_report/en/.

3. Instituto Nacional de Enfermedades Respiratorias "Dr. Emilio Coni". Notificación de casos de tuberculosis en la República Argentina, período 1980-2015. Santa Fe: Ministerio de Salud - ANLIS; 2016. [Acceso: 3 de febrero de 2017]. Disponible en: http:/ / www.anlis.gov. ar/iner/wp-content/uploads/2016/11/ Notificacionde-casos-de-Tuberculosis-en-la-Republica-ArgentinaPeriodo-1980-2015.pdf.

4. Comité Nacional de Neumonología; Comité Nacional de Infectología. Criterios de diagnóstico y tratamiento de la tuberculosis infantil. Resumen ejecutivo. Arch Argent Pediatr 2016; 114(2):189-90.

5. Mohapatra PR, Janmeja AK. Tuberculous Lymphadenitis. J Assoc Physicians India 2009; 57:585-90.

6. Nelson L, Schneider E, Wells Ch, et al. Epidemiology of childhood tuberculosis in the United States, 1993-2001: The need for continued vigilance. Pediatrics 2004; 114(2):333-41.

7. Handa U, Mundi I, Mohan S. Nodal tuberculosis revisited: a review. J Infect Dev Ctries 2012; 6(1):6-12.

8. Geldmacher H, Taube C, Kroeger C, et al. Assessment of lymph node tuberculosis in northern Germany: a clinical review. Chest 2002; 121(4):1177-82.

9. Fain $\mathrm{O}$, Lortholary $\mathrm{O}$, Djouab $\mathrm{M}$, et al. Lymph node tuberculosis in the suburbs of Paris: 59 cases in adults not infected by the human immunodeficiency virus. Int JTuberc Lung Dis 1999; 3(2):162-5.

10. Perlman DC, D'Amico R, Salomon N. Mycobacterial Infections of the Head and Neck. Curr Infect Dis Rep 2001; 3(3):233-41.

11. Kamal MS, Hoque MH, Chowdhury FR, et al. Cervical Tuberculous Lymphadenitis: Clinico-demographicProfiles of Patients in a Secondary Level Hospital of Bangladesh. Pak J Med Sci 2016; 32(3):608-12.

12. Chen YM, Lee PY, Su WJ, et al. Lymph node tuberculosis: 7-year experience in Veterans General hospital, Taipei, Taiwan. Tuber Lung Dis 1992; 73(6):368-71.

13. Purohit MR, Mustafa T, MorkveO, etal.Gender differences in the clinical diagnosis of tuberculous lymphadenitis-a hospital-based study from Central India. Int J Infect Dis 2009; 13(5):600-5.

14. Khan FY. Clinical pattern of tuberculous adenitis in Qatar: experience with 35 patients. Scand J Infect Dis 2009;41(2):12834.

15. Penfold CN, Revington PJ. A review of 23 patients with 
tuberculosis of the head and neck. Br J Oral Maxillofac Surg 1996; 34(6):508-10.

16. Polesky A, Grove W, Bhatia G. Peripheral tuberculous lymphadenitis: epidemiology, diagnosis, treatment, and outcome. Medicine (Baltimore) 2005; 84(6):350-62.

17. Song KH, Jeon JH, Park WB, et al. Usefulness of the wholeblood interferon-gamma release assay for diagnosis of extrapulmonary tuberculosis. Diagn Microbiol Infect Dis 2009; 63(2):182-7.

18. Khan R, Harris SH, Verma AK, et al. Cervical lymphadenopathy: scrofula revisited. J Laryngol Otol 2009; 123(7):764-7.

19. Starke J, Muñoz F. Tuberculosis. In: Berhman R, Kliegman RM, Jenson HB, eds. Nelson Textbook of Pediatrics. 16th ed. London: W. B. Saunders Co; 2000.P.885-95.

20. Ibekwe AO, al Shareef $Z$, al Kindy S. Diagnostic problems of tuberculous cervical adenitis (scrofula). Am JOtolaryngol 1997; 18(3):202-5.

21. Jha BC, Dass A, Nagarkar NM, et al. Cervical tuberculous lymphadenopathy: Changing clinical pattern and concepts in management. Postgrad Med J 2001; 77(905):185-7.

22. Magsi PB, Jamro B, Shaikh AA, et al. An audit of 140 cases of cervical lymphadenopathy at tertiary care hospital. Gomal J Med Sci 2013; 11(1):47-9.

23. Choudhury N, Bruch G, Kothari P, et al. 4 years' experience of head and neck tuberculosis in a south London hospital. $J$ R Soc Med 2005; 98(6):267-9.

24. HowieS, Voss L, Baker L, et al. Tuberculosis in New Zealand, 1992-2001: a resurgence. Arch Dis Child 2005; 90(1):1157-61.

25. Yates MD, GrangeJM. Bacteriological survey of tuberculous lymphadenitis in southeast England, 1981-1989.J Epidemiol Community Health 1992; 46(4):332-5.

26. Kazwala RR, Daborn CJ, Sharp JM, et al. Isolation of Mycobacterium bovis from human cases of cervical adenitis in Tanzania: a cause for concern? Int J Tuberc Lung Dis 2001; 5(1):87-91.
27. Ellison E, Lapuerta P, Martin SE. Fine needle aspiration diagnosis of mycobacterial lymphadenitis. Sensitivity and predictive value in the United States. Acta Cytol 1999; 43(2):153-7.

28. Wright CA, van der Burg M, Geiger D, et al. Diagnosing mycobacterial lymphadenitis in children using fine needle aspiration biopsy: cytomorphology, ZN staining and autofluorescence-making more of less. Diagn Cytopathol 2008; 36(4):245-51.

29. Chand P, Dogra R, Chauhan N, et al. Cytopathological Pattern of Tubercular Lymphadenopathy on FNAC: Analysis of 550 Consecutive Cases. J Clin Diagn Res 2014; 8(9):FC16-9.

30. Cho OH, Park KH, Kim T, et al. Paradoxical responses in non-HIV-infected patients with peripheral lymph node tuberculosis. J Infect 2009; 59(1):56-61.

31. Hawkey CR, Yap T, Pereira J, et al. Characterization and management of paradoxical upgrading reactions in HIVuninfected patients with lymph node tuberculosis. Clin Infect Dis 2005; 40(9):1368-71.

32. Campbell IA, Ormerod LP, Friend JA, et al. Six months versus nine months chemotherapy for tuberculosis of lymph nodes: final results. Respir Med 1993; 87(8):621-3.

33. Yuen AP, Wong SH, Tam CM, etal. Prospective randomized study of thrice weekly six-month and nine-month chemotherapy for cervical tuberculous lymphadenopathy. Otolaryngol Head Neck Surg 1997; 116(2):189-92.

34. Van Loenhout-Rooyackers JH, Laheij RJ, Richter C, et al. Shortening the duration of treatment for cervical tuberculous lymphadenitis. Eur Respir J 2000; 15(1):192-5.

35. Nahid P, Dorman SE, Alipanah N, et al. Official American Thoracic Society/Centers for Disease Control and Prevention/Infectious Diseases Society of America Clinical Practice Guidelines: Treatment of Drug-Susceptible Tuberculosis. Clin Infect Dis 2016; 63(7):e147-95. 